\title{
Porosity Characterization of Thermal Barrier Coatings by Ultrasound with Genetic Algorithm Backpropagation Neural Network
}

\author{
Shuxiao Zhang $\mathbb{D}^{1,},{ }^{1,2,3,4,5}$ Gaolong $L v \mathbb{D}^{1,2,3,4,5}$ Shifeng Guo $\mathbb{D}^{1,2,3,4,5}$ Yanhui Zhang $\mathbb{D}^{1,2,3,4,5}$ \\ and Wei Feng $\mathbb{D}$ 1,2,3,4,5
}

${ }^{1}$ CAS Key Laboratory of Human-Machine Intelligence-Synergy Systems, Shenzhen Institutes of Advanced Technology, Chinese Academy of Sciences, Shenzhen 518055, China

${ }^{2}$ Guangdong Ultrasonic Nondestructive Testing Engineering Technology Research Center, Chinese Academy of Sciences, Shenzhen 518055, China

${ }^{3}$ Shenzhen Key Laboratory of Smart Sensing and Intelligent Systems, Shenzhen Institutes of Advanced Technology, Chinese Academy of Sciences, Shenzhen 518055, China

${ }^{4}$ Guangdong Provincial Key Lab of Robotics and Intelligent System, Shenzhen Institutes of Advanced Technology, Chinese Academy of Sciences, Shenzhen 518055, China

${ }^{5}$ Shenzhen College of Advanced Technology, University of Chinese Academy of Sciences, Beijing, China

Correspondence should be addressed to Wei Feng; wei.feng@siat.ac.cn

Received 7 August 2020; Revised 1 September 2020; Accepted 20 April 2021; Published 29 April 2021

Academic Editor: Átila Bueno

Copyright ( $\odot 2021$ Shuxiao Zhang et al. This is an open access article distributed under the Creative Commons Attribution License, which permits unrestricted use, distribution, and reproduction in any medium, provided the original work is properly cited.

\begin{abstract}
Porosity is considered as one of the most important indicators for the characterization of the comprehensive performance of thermal barrier coatings (TBCs). In this study, the ultrasonic technique and the artificial neural network optimized with the genetic algorithm (GA_BPNN) are combined to develop an intelligent method for automatic detection and accurate prediction of TBCs's porosity. A series of physical models of plasma-sprayed $\mathrm{ZrO}_{2}$ coating are established with a thickness of $288 \mu \mathrm{m}$ and porosity varying from $5.71 \%$ to $26.59 \%$, and the ultrasonic reflection coefficient amplitude spectrum (URCAS) is constructed based on the time-domain numerical simulation signal. The characteristic features $\left(f_{1}, f_{2}, A_{\max }, \Delta A\right)$ of the URCAS, which are highly dependent on porosity, are extracted as input data to train the GA_BPNN model for predicting the unknown porosity. The average error of the prediction results is $1.45 \%$, which suggests that the proposed method can achieve accurate detection and quantitative characterization of the porosity of TBCs with complex pore morphology.
\end{abstract}

\section{Introduction}

Thermal barrier coatings (TBCs) are widely used in the aerospace industry to protect critical components from wear, erosion, corrosion, or thermal oxidation [1]. The thermal insulation performance of TBCs as well as their strain compliance required to accommodate the thermal expansion coefficient mismatch between metal and ceramic upper layers strongly depends on its porosity. Porosity can significantly affect the comprehensive performance of TBCs $[2,3]$. Hence, how to effectively characterize the microstructure is a mandatory requirement for optimizing TBCs performance, monitoring service life, and developing new TBCs $[4,5]$. Nondestructive testing (NDT) technology has been considered as an ideal solution.

Numerous nondestructive methods have been proposed for quantitative determination of porosity in TBCs, such as thermography method [6-10], terahertz technique [11], $\mathrm{X}$-ray [12], and effective medium theory [13]. Among all existing methods, the ultrasonic technique is a relatively inexpensive, fast, and reliable nondestructive method, which has been widely used to detect porosity in thermally sprayed 
coatings [3, 14-17]. A representative method is to use ultrasonic parameters in thermally sprayed coatings, usually longitudinal wave velocity and ultrasonic attenuation, to evaluate its properties, including porosity $[15,17-20]$. For example, a trend line or corresponding expression between the velocity/attenuation and porosity for the coatings investigated is used to estimate the porosity content of other parts with the same coatings by measuring their thickness and velocity $[17,19]$. However, the relationship between ultrasonic characteristic parameters and coating porosity is complex and nonlinear, and there are many uncertainties in predicting porosity. And the process of establishing a fitting curve or empirical formula can be an expensive and timeconsuming process. A recent breakthrough in computation and artificial intelligence provides a new solution for porosity prediction.

The machine learning method has been proved to be a powerful tool to deal with complex multiparameter and nonlinear problems, especially for dealing with highly nonlinear noise and incomplete data sets [21-23]. In recent years, machine learning approaches such as artificial neural networks (ANNs) have been widely used in the nondestructive testing area [24-26], especially for the porosity characterization of thermal barrier coatings. Li et al. [27] used infrared thermal images and the algorithm that combines gray gradient space histogram entropy and sparse representation-based classifier to detect the porosity. Ye et al. [28] implemented a novel hybrid method based on the support vector machine algorithm optimized by the cuckoo search algorithm (CS-SVM) for predicting microstructural features of thermal barrier coatings using various process parameters, such as porosity. Ye et al. [29] established a novel approach based on terahertz time-domain spectroscopy combined with principal component analysis support vector machine (PCA-SVM) to characterize microstructural features of thermal barrier coatings such as porosity, poreto-crack ratio, and pore size. The experimental results revealed that the proposed method can be implemented for characterizing microstructural features efficiently. The above work shows that machine learning can be used to characterize the porosity of thermal barrier coatings by combining infrared thermal technology, terahertz technology, and various processing parameters. At the same time, machine learning has also been applied in the field of ultrasound. Timo et al. [30] proposed a convolution neural network to evaluate the change of feasible data of ultrasonic tomography to determine the porosity and curvature of porous materials, and the method is feasible. However, the absolute error is higher than $10 \%$ for the low porosity medium. Ma et al. [31] proposed a hybrid method that combines the BP neural network optimizing Gaussian process regression algorithm and the ultrasonic technique and can be used to characterize porosities of thermal barrier coatings. Li et al. [32] developed a characterization method based on a support vector machine model based on the particle swarm optimization algorithm (PSO-SVR) to predict the porosity of the AlSi-polyester seal coating. The experimental results suggest that ultrasonic attenuation coefficients obtained by continuous wavelet transform rely on porosity. However, the above work requires complex signal process methods to extract characteristic parameters for the training network model, which is a time-consuming process.

In this study, a new method is proposed to quantitatively characterize the porosity of thermal barrier coatings by using a machine learning algorithm and ultrasonic technology. First, the ultrasonic numerical simulation model is established to simulate the interaction of ultrasonic waves with different porosities of thermal barrier coatings. Next, the BP neural network model optimized by the GA algorithm is proposed, and the characteristic parameters in the time and frequency domain of ultrasonic responses, which are dependent on the porosities, are extracted as inputs, whereas the porosity of thermal barrier coatings is used as outputs. Finally, the feasibility and validity of the developed GABPNN approach are verified.

\section{Numerical Simulation Method}

2.1. Detection Method and Characteristic Parameter Extraction. Figure 1 shows the schematic diagram of the wave propagating perpendicularly in the three-media structure consisting of water $\left(Z_{1}\right)$ /coating $\left(Z_{2}\right)$ /substrate $\left(Z_{3}\right)$. A broadband longitudinal wave propagates in it and generates an infinite series of acoustic reflection and transmission signals. Because the TBCs are usually tens to hundreds of microns in thickness, the echoes reflected from the sequence interfaces usually overlap. It is difficult to directly extract the time-domain characteristics. In this paper, a normalized URCAS technique is utilized to obtain the frequency-domain characteristic.

The frequency dependence of the reflection coefficient $R(f)$ of the coating can be written as $[14,33]$

$$
\begin{aligned}
R(f) & =\sqrt{\operatorname{Re}^{2}+I^{2}} \\
& =\left[\frac{\left(r_{12}+r_{23} \exp (-2 \partial d)\right)^{2}-4 r_{12} r_{23} \exp (-2 \partial d) \sin ^{2}(2 \pi f d / c)}{\left(1+r_{12} r_{23} \exp (-2 \partial d)\right)^{2}-4 r_{12} r_{23} \exp (-2 \partial d) \sin ^{2}(2 \pi f d / c)}\right]^{1 / 2},
\end{aligned}
$$

where $r_{12}$ and $r_{23}$ are the reflection coefficients of the interfaces between coupling medium 1/coating $2 /$ substrate 3 , respectively. $d, \alpha$, and $c$ are the coating thickness, ultrasonic attenuation coefficient, and longitudinal wave velocity of the coating, respectively.

A series of extreme values of URCAS will be reached when $\sin (4 \pi f d / c)=0$, that is,

$$
f_{n}=\frac{n c}{4 d}
$$

where $f_{n}$ is the harmonic frequency.

The longitudinal wave velocity of a material depends on Young's modulus and the density of the material, and its expression is

$$
c=\sqrt{\frac{E}{\rho}}
$$

where $E$ is Young's modulus and $\rho$ is the density of the material. 


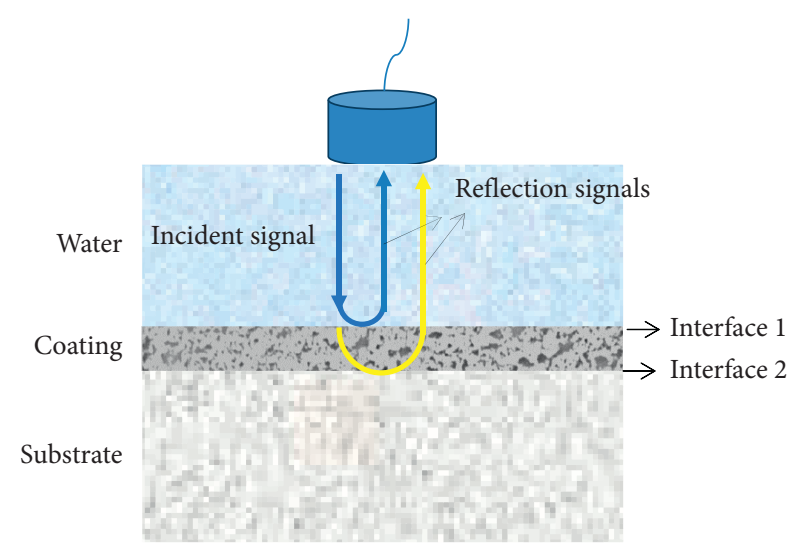

Figure 1: The diagram of ultrasonic propagation in a water/ coating/substrate system.

It can be found from equations (1)-(3) that the density and elastic modulus of the coating change due to the existence of pores in the coating, which leads to the change of longitudinal wave sound velocity, reflection, and attenuation coefficient of the material and finally leads to the change of resonance frequency $f_{n}$ and the corresponding amplitude in the amplitude spectrum of sound pressure reflection coefficient with the change of porosity [34, 35]. Therefore, the amplitude corresponding to the minimum and maximum of resonant frequency and the amplitude difference between the minimum and maximum of resonant frequency can be extracted as characteristic parameters [36] and as input data to train the GA_BPNN model, as shown in Table 1 .

2.2. SEM In Situ Modeling Theory. Even if the porosity of the coating is determined, due to the different morphology of pores in different positions, the local density and Young's modulus of the coating will fluctuate to some extent, which will also cause the fluctuation of ultrasonic velocity [18]. Therefore, $\mathrm{P}$-wave velocity is a multivariate function of porosity, pore morphology, pore distribution, and so on. To avoid the introduction of multiple variables, this paper is different from the traditional regular pore model and random medium model and adopts an in situ SEM photo modeling method that preserves the actual morphology and distribution of pores in $\mathrm{ZrO}_{2}$ coating. A series of geometric models with the same pore morphology and pore distribution but different porosities are constructed based on SEM photos and image processing software. After the geometric model of materials is established, the model can be used to study the ultrasonic properties of different materials by assigning different elastic parameters to each region.

$\mathrm{ZrO}_{2}$ belongs to the cubic system and has three independent elastic constants: $C_{11}, C_{12}$, and $C_{44}$. According to the law of elasticity, the relationship between stress and strain can be written as
TABle 1: Frequency-domain characteristic parameters.

\begin{tabular}{lccc}
\hline Parameters 1 & Parameters 2 & Parameters 3 & Parameters 4 \\
\hline$f_{1}$ & $f_{2}$ & $R\left(f_{1}\right)$ & $R\left(f_{1}\right)-R\left(f_{2}\right)$ \\
\hline
\end{tabular}

$$
\left[\begin{array}{c}
\sigma_{11} \\
\sigma_{22} \\
\sigma_{33} \\
\sigma_{12} \\
\sigma_{23} \\
\sigma_{31}
\end{array}\right]=\left[\begin{array}{cccccc}
C_{11} & C_{12} & C_{12} & 0 & 0 & 0 \\
C_{12} & C_{11} & C_{12} & 0 & 0 & 0 \\
C_{12} & C_{12} & C_{11} & 0 & 0 & 0 \\
0 & 0 & 0 & C_{44} & 0 & 0 \\
0 & 0 & 0 & 0 & C_{44} & 0 \\
0 & 0 & 0 & 0 & 0 & C_{44}
\end{array}\right]\left[\begin{array}{c}
\varepsilon_{11} \\
\varepsilon_{22} \\
\varepsilon_{33} \\
2 \varepsilon_{12} \\
2 \varepsilon_{23} \\
2 \varepsilon_{31}
\end{array}\right],
$$

where $\sigma$ is stress and $\varepsilon$ is strain. From the wave equation, the elastic constant of the material and the density of the material are determined, and the sound velocity of the material can be calculated. In this paper, the microstructure characteristics of $\mathrm{ZrO}_{2}$ coating are studied, and the Hudson model used in the geophysical survey is used to modify the elastic parameters of $\mathrm{ZrO}_{2}$. The parameters required in the numerical calculation are shown in Table 2 [37].

\subsection{Construction of Pore Models with Different Porosities in} $\mathrm{ZrO}_{2}$ Coating. According to the in situ modeling principle of SEM photos, a series of 24 physical models with the same pore morphology and pore distribution but different porosities are constructed. The porosity of the physical model varies from $5.7 \%$ to $26.59 \%$, covering $7 \%$ to $25 \%$ of the porosity of common ceramic coatings. The physical models constructed by this method have the following advantages: (1) compared with other methods, they are similar to the actual morphology of pores in $\mathrm{ZrO}_{2}$ coating, which makes the calculation more reliable; (2) pore morphologies of different porosity models are equivalent. Following the principle of controlling a single variable, the longitudinal wave velocity only changes with porosity, and the research results are more rigorous.

For the physical model of $\mathrm{ZrO}_{2}$ coating, the ultrasonic numerical simulation is carried out by the finite difference time-domain method (FDTD). The main parameters used in the simulation are shown in Table 3.

The pulse-echo reflection method is used to simulate the narrow pulse sound source with a frequency of $5 \mathrm{MHz}$. The waveform of the sound source is shown in Figure 2, and the physical model used in the simulation is shown in Figure 3.

\section{Artificial Neural Networks and GA-BPNN}

3.1. Artificial Neural Networks. ANN generally acquires knowledge by training on a lot of input data and stores the information in the weights and biases of the neural networks and transmits information through forwarding propagation through the networks [38]. Backpropagation neural network (BPNN) is first proposed by Rumelhart et al. in 1985, which is the improvement of ANN algorithm learning representation 
TABLE 2: Material parameters in numerical calculation of ultrasonic testing.

\begin{tabular}{lccccc}
\hline Medium & $C_{11}(\mathrm{MPa})$ & $C_{12}(\mathrm{MPa})$ & $C_{44}(\mathrm{MPa})$ & Density $\left(\mathrm{kg} \cdot \mathrm{m}^{-3}\right)$ & 6500 \\
\hline $\mathrm{ZrO}_{2}$ coating & 142740 & 70039 & 36200 & $V_{L}(\mathrm{~m} / \mathrm{s})$ \\
$20^{\circ} \mathrm{C}$ air & 0.1467 & 0.1467 & 0 & 1.24 & 2957.65 \\
\hline
\end{tabular}

TABle 3: Parameter and value used for ultrasonic numerical simulation.

\begin{tabular}{llll}
\hline Center frequency & $5 \mathrm{MHz}$ & Velocity of water & $1497 \mathrm{~m} / \mathrm{s}$ \\
\hline Thickness of the coating & $288 \mu \mathrm{m}$ & Velocity of $\mathrm{ZrO}_{2}$ & $2957.65 \mathrm{~m} / \mathrm{s}$ \\
\hline
\end{tabular}

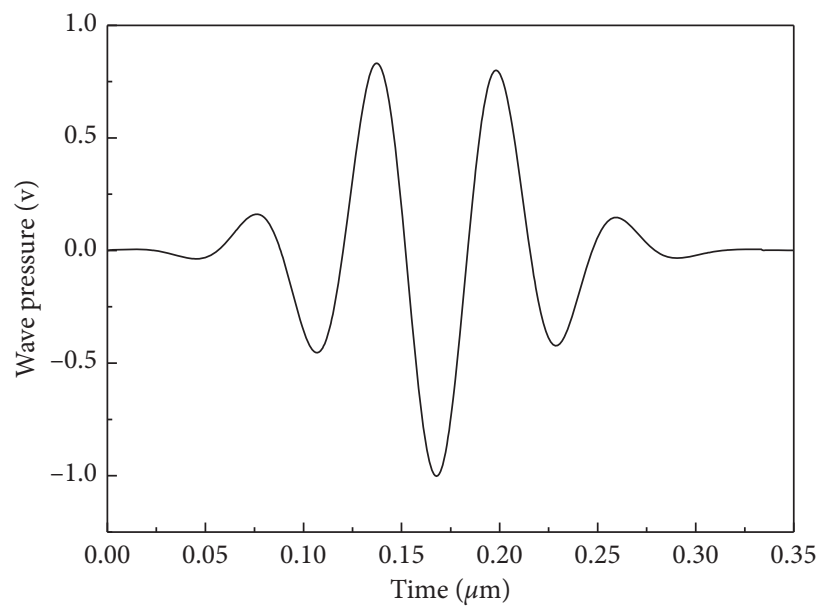

FIgURE 2: Source wave used in the simulation.

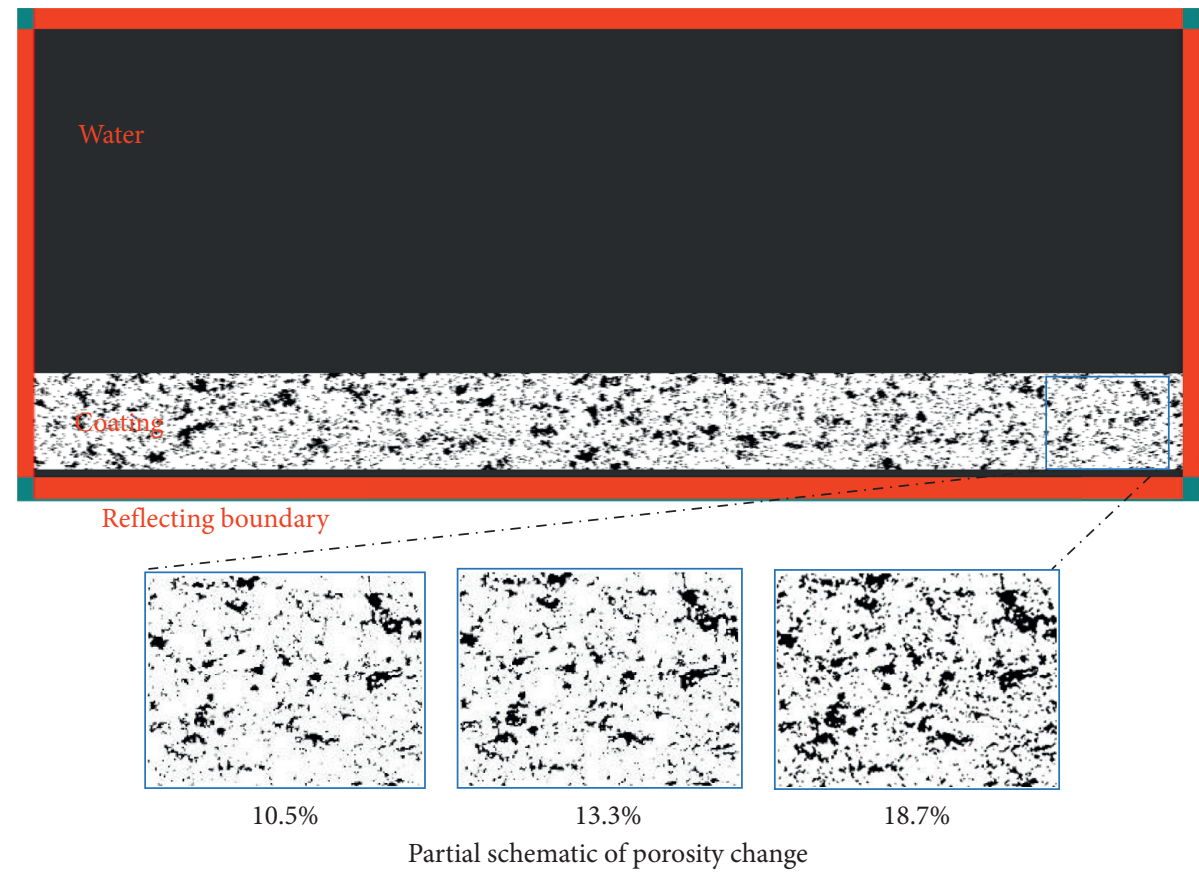

Figure 3: Physical model used in the simulation.

by backpropagating errors [39]. Generally, a BPNN model consists of three layers, including one input layer, at least one hidden layer, and one output layer. The neuron is the basic structure unit of neural network models, which distributes in different layers, and can be highly interconnected with other neural in adjacent layers according to a certain topological structure. Figure 4 shows a three-layer architecture of BPNN, with the flow of the information presented. 


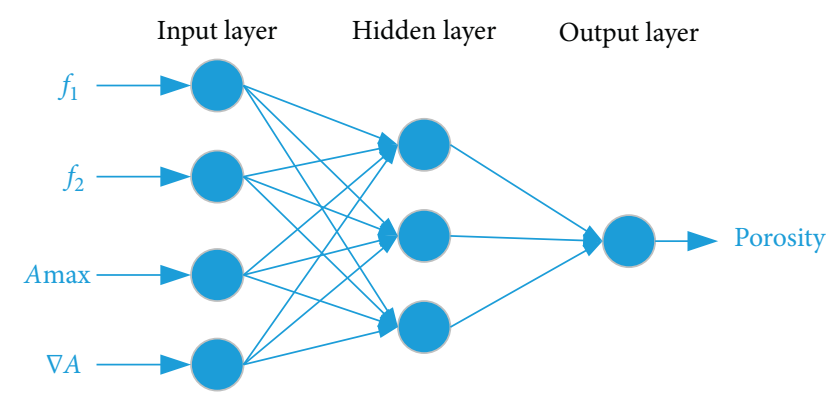

FIgURE 4: A three-layer architecture of BPNN.

3.2. GA-BPNN Method. A multi-layered BPNN model with appropriate configuration enables the solution of complex systems. However, some disadvantages still exist according to the random selection of initial weights and thresholds of the BPNN model, such as overfitting and local minima problems, which can mitigate the accuracy of predicted results [40]. Bayesian regularization algorithm is adopted as the training algorithm to overcome the shortcoming of overfitting [41]. Meanwhile, an improved genetic algorithm backpropagation neural network (GA-BPNN) model is implemented to optimize the parameters and topology of the BPNN model and to avoid local convergence and obtain accurate solution [42]. The genetic algorithm with global stochastic search capacity is applied to optimize the original biases and weights of BPNN to help BPNN converge more quickly and find an accurate solution [43].

The main operation of the genetic algorithm can be summarized as follows [44]: (i) randomly generate an initial population with several individuals and (ii) evaluate the fitness value of all individuals with the predefined fitness function (mean absolute percentage error, MAPE) [45]. Individuals with high fitness values are prone to produce the next generation. (iii) Selection operation, crossover operation, and mutation operation are implemented to generate subgeneration of individuals from existing individuals in the population. (iv) Repeat steps (ii) and (iii) until the optimum individual is obtained. Figure 5 shows the flow chart of the BPNN model optimized with the genetic algorithm. Firstly, the initial population that can be encoded by the initial values of weights and biases is used to evaluate the fitness value of all individuals. Then, the genetic algorithm will select high fitness individuals and obtain a better subpopulation. After meeting the requirements, the process of training will stop and optimal individuals are decoded to weights and biases of the BPNN model.

\section{Results and Discussion}

4.1. Simulated Results. Figure 6 shows time-domain signals of the numerical simulation, which are obtained when the coating porosity is $5.71 \%$ minimum and $26.59 \%$ maximum, respectively. It can be seen from the figure that the reflection echo from the bottom of the coating with the coating porosity characteristics is submerged in the surface wave, which cannot be directly analyzed effectively. The ultrasonic reflection coefficient amplitude spectroscopy of coating under different porosity is obtained by spectrum analysis of the time-domain signal, shown in Figure 7. It is observed that the resonance frequency of the ultrasonic reflection coefficient amplitude spectroscopy of coating with larger porosity moves towards the low-frequency band, and the higher the porosity, the greater the deviation. At the same time, the amplitude of normalized amplitude spectrum also varies with porosity.

4.2. Feature Selection. Sequentially read characteristic parameters such as resonance frequency maximum value $f_{1}$, resonance frequency minimum value $f_{2}$, amplitude $A_{\max }$ corresponding to $f_{1}$, and amplitude difference $(\Delta A)$ corresponding to $f_{1}$ and $f_{2}$ from Figure 7 . The normalized characteristic parameters are shown in Figure 8. It is observed that the normalized amplitude of extracted features varies with porosity, which indicates that after normalization, the extracted features from ultrasonic signals are a function of porosity.

To ensure the accuracy and robustness of the neural network, 5 varying levels of Gaussian random noise $(1 \%, 2 \%$, $3 \%, 4 \%$, and $5 \%$ relative to the original data) are added to the features, for expanding the data sets.

4.3. GA-BPNN Training and Testing. In this paper, the GABPNN model with three layers has been proposed to predict unknown porosities of thermal barrier coatings. A trial anderror method is implemented to determine the optimal structure of the network model, and the network structure that is adjusted repeatedly until attaining optimal performance, which can mitigate overfitting to a great extent. As shown in Figure 4, the optimal structure of the GA-BPNN model is set to 4-3-1 (four neurons in the input layer, three neurons in the hidden layer, and one neuron in the output layer). The four parameters $\left(f_{1}, f_{2}, A_{\max }, \Delta A\right)$ are selected as input variables of the network model, and the porosity of different thermal barrier coatings is the output of the model. Table 4 shows the configuration parameters of the GA-BPNN model for predicting the porosity of thermal barrier coatings.

After the GA-BPNN model has been trained with various input features extracted from different thermal barrier coatings, it can not only enable the prediction of different porosities but also mitigate the time of complex solution to a great extent. Once the four extracted features are fed to the model, the porosity is predicted as the output of the network. To ensure the robustness of the neural network and acquire better performance of predicting the porosity, the signal of each porosity of thermal barrier coatings is contaminated by five levels of Gaussian random noise $(1 \%, 2 \%, 3 \%, 4 \%$, and $5 \%$ of the original data) before the training process. Therefore, the total number of 132 sets of data is obtained from the original data and noise-contaminated data. Each set of total sets contains four extracted sensitive features $\left(f_{1}, f_{2}, A_{\max }, \Delta A\right)$ as the input of the GA-BPNN model, and one measured parameter (porosity of thermal barrier coatings) as the output of the model or the target to verify the prediction of the network model. 


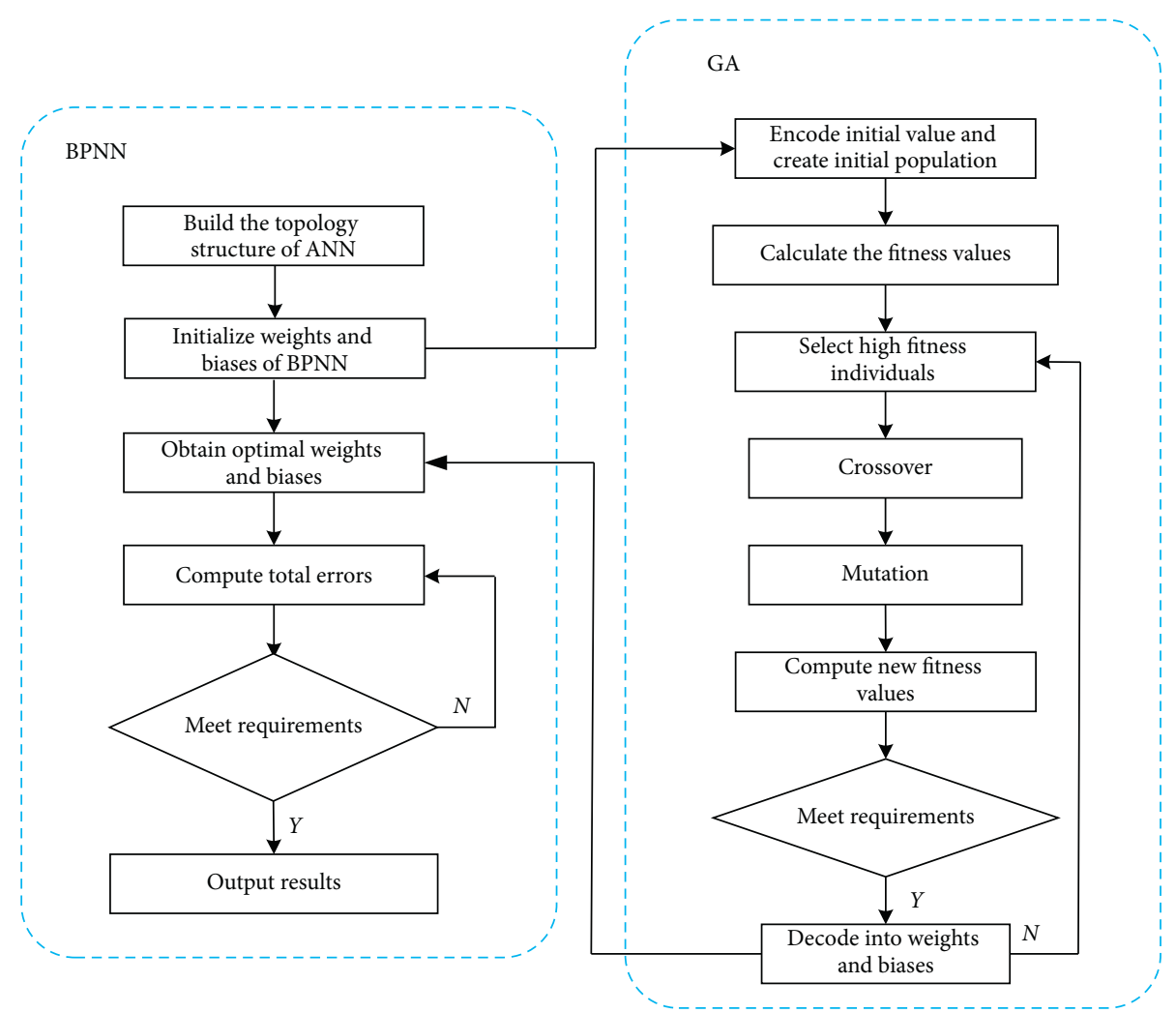

Figure 5: The flow chart of the GA_BPNN model.

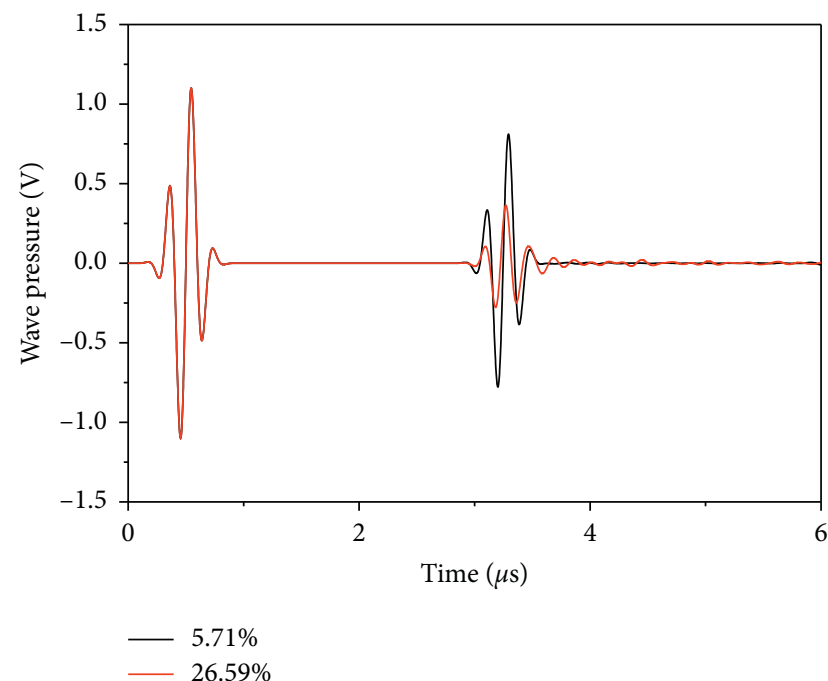

FIgURE 6: Simulation signals of coatings with 5.71\% minimum and $26.59 \%$ maximum, respectively.

The 132 data sets are randomly divided into 117 training data sets to train the network model to acquire the optimized weights and biases and ultimately ensure the accuracy of the model and 15 sets of data which are implemented to identify the performance of the trained network.

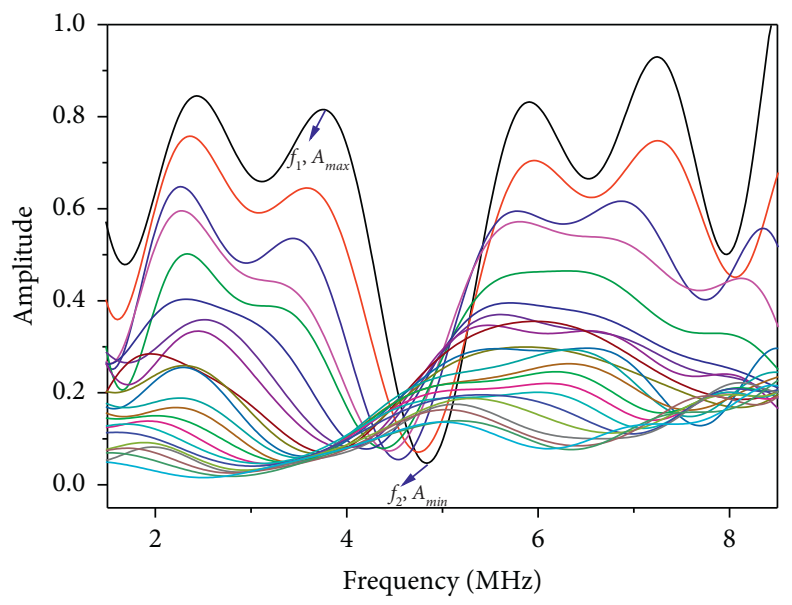

Porosity (\%)

$\begin{array}{lll}-5.71 & -15.38 & -21.3 \\ 7.16 & -16.31 & -22.1687 \\ 8.39 & -17.0224 & -23.04 \\ -9.32 & -17.9 & -23.9057 \\ 10.5 & -18.7475 & -24.78 \\ -12.09 & -19.6 & -25.6762 \\ 13.31 & -20.4492 & -26.59 \\ 14.39 & & \end{array}$

FIgURE 7: Numerical simulation results of the amplitude spectrum of the sound pressure reflection coefficient of $\mathrm{ZrO}_{2}$ coating with different porosity. 


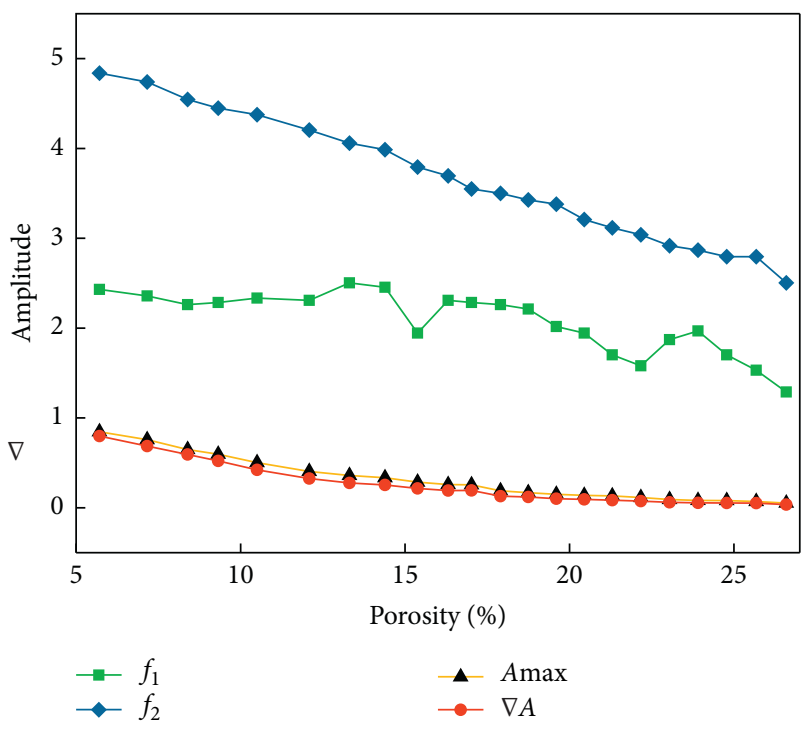

FIGURE 8: Variation of characteristic parameters with porosity.

TABLE 4: The configuration parameters of the GA-BPNN model.

\begin{tabular}{lcc}
\hline Name & Parameters & Value \\
\hline \multirow{3}{*}{ BPNN } & Learning rate & 0.0015 \\
& Training goal & 0.0001 \\
& Epoch & 1000 \\
& Training function & Trainlm \\
& Transfer function & Sigmoid \\
\hline \multirow{3}{*}{ GA } & Cycle index & 10000 \\
& Maxgen & 60 \\
& Selectivity factor & 1.0 \\
& Crossover factor & 0.3 \\
& Mutation factor & 0.1
\end{tabular}

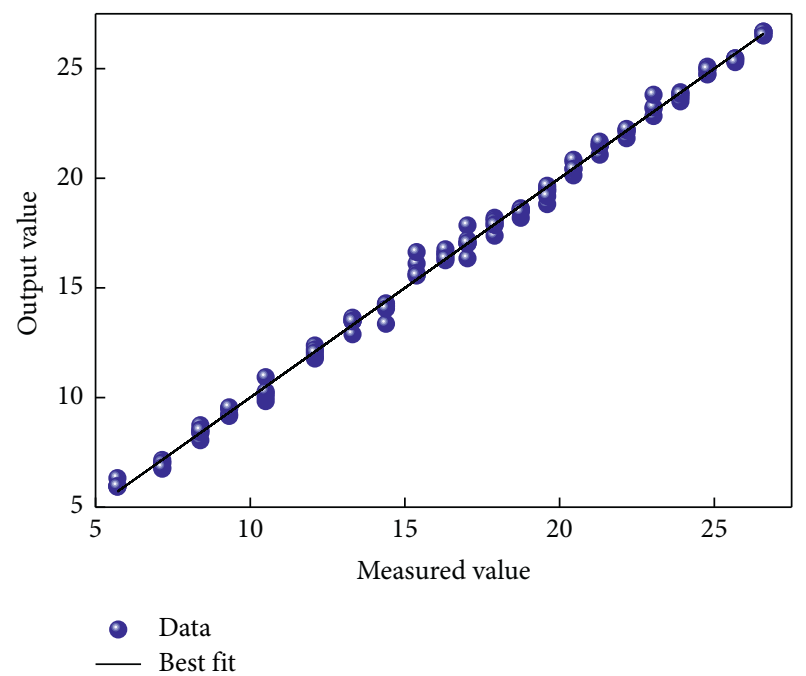

Figure 9: Comparison of output values in the training process versus the corresponding measured values.

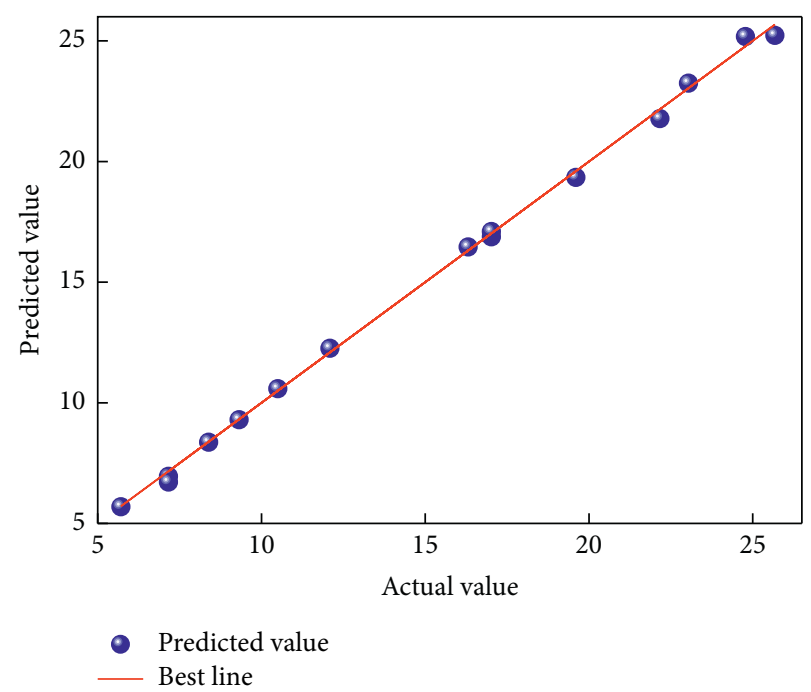

FIGURE 10: Comparison of actual value and predicted value of the testing process.

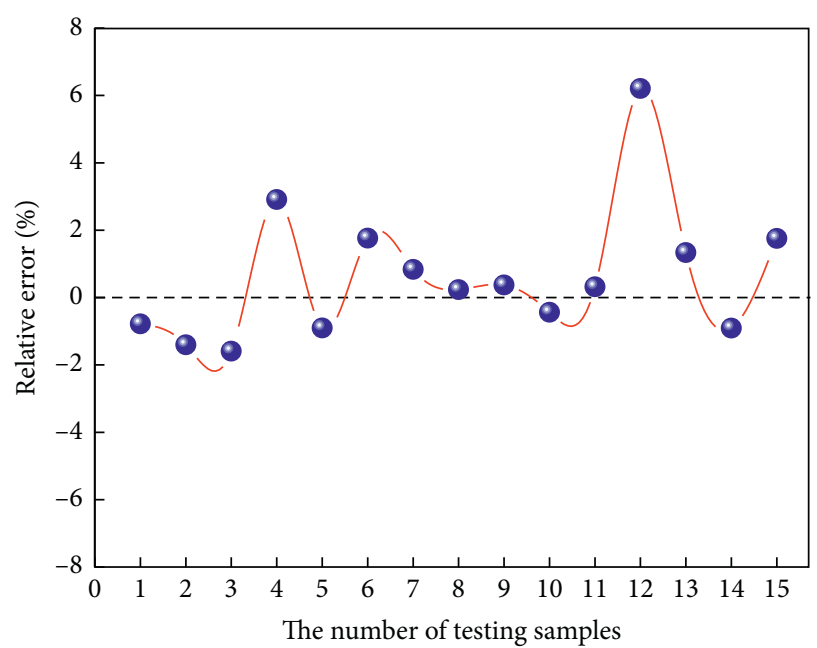

FIGURE 11: The relative error of the actual value and predicted value.

4.4. Porosity Prediction Results and Discussion. After the training process, Figure 9 shows the training effects of the GA-BPNN model using 117 training data sets. It shows that all data follow the best fit line, which indicates that there is good consistency between the output value and the measured value. Then, according to the well-trained GA-BPNN model, the remaining 15 testing data sets are implemented to verify the performance of the network.

Figure 10 shows the predicted porosities of the thermal barrier coatings using the proposed GA-BPNN method. It can be seen that the predicted values of porosity are in good agreement with the measured values of porosity. In this case, the relative errors of the proposed method are all less than 
$6.2 \%$ as shown in Figure 11 and the average of the errors is $1.45 \%$, which indicates that the proposed method can accurately predict the porosities. Therefore, the extracted characteristic features are implemented to train the proposed GA-BPNN model for quantitatively evaluating the size of porosity, which is reliable and feasible.

\section{Conclusion}

In this paper, a hybrid nondestructive testing method is proposed to detect and quantitatively characterize the porosity of thermal barrier coatings using the ultrasonic technique and neural networks. The artificial neural network optimized with the genetic algorithm (GA-BPNN) is developed, with the SEM in situ model simulation offering sufficient ultrasonic signals for training and testing. A total number of 132 data sets are used to train and test the performance of the GA_BPNN model. The porosity prediction error is less than $6.2 \%$ and the average error is $1.45 \%$. The results show that the hybrid nondestructive testing method, which combines ultrasonic technology, numerical simulation, and genetic BPNN model, is proved to be a powerful tool to characterize the porosity of porous materials and can be used to develop a neural network model to automatically characterize the porosity of thermal barrier coatings.

\section{Data Availability}

The data used to support the findings of this study are available from the corresponding author upon request.

\section{Conflicts of Interest}

The authors declare that they have no conflicts of interest.

\section{Authors' Contributions}

Shuxiao Zhang and Gaolong Lv contributed equally to this work.

\section{Acknowledgments}

The authors are grateful to the financial support from the Key-Area R\&D Program of Guangdong Province (no. 2020B090925002), Science and Technology Innovation Commission of Shenzhen (no. ZDSYS20190902093209795), Science, Technology and Fundamental Research and Discipline Layout project of Shen Zhen (no. JCYJ20170818153048647), and the joint Research Fund between the National Natural Science Foundation of China and Shenzhen (no. U1813222).

\section{References}

[1] A. Fahr, S. Giguere, B. Roge, K. McRae, and S. Safizadeh, "Application of NDE in aerospace coatings," in Proceedings of the Smart Nondestructive Evaluation for Health Monitoring of Structural and Biological Systems. International Society for Optics and Photonics, pp. 83-92, Portland, OR, USA, March 2002.
[2] D. Ye, W. Wang, H. Zhou et al., "In-situ evaluation of porosity in thermal barrier coatings based on the broadening of terahertz time-domain pulses: simulation and experimental investigations," Optics Express, vol. 27, no. 20, pp. 28150-28165, 2019.

[3] S. Parthasarathi, B. Tittmann, and E. J. Onesto, "Ultrasonic technique for measuring porosity of plasma-sprayed alumina coatings," Journal of Thermal Spray Technology, vol. 6, pp. 486-488, 1997.

[4] L. B. Chen, "Yttria-stabilized zirconia thermal barrier coatings-a review," Surface Review and Letters, vol. 13, pp. 535-544, 2006.

[5] D. A. Luo, E. T. Barraza, and M. W. Kudenov, "Mueller matrix polarimetry on plasma sprayed thermal barrier coatings for porosity measurement," Applied Optics, vol. 56, no. 35, pp. 9770-9778, 2017.

[6] F. J. S. Cernuschi and C. Technology, "Can TBC porosity be estimated by non-destructive infrared techniques?" A Theoretical and Experimental Analysis, vol. 272, pp. 387-394, 2015.

[7] D. C. Jiao, W. X. Shi, Z. W. Liu, and H. M. Xie, "Laser multimode scanning thermography method for fast inspection of micro-cracks in TBCs surface," Journal of Nondestructive Evaluation, vol. 37, p. 10, 2018.

[8] M. F. Akbar, G. N. Jawad, C. I. Duff, and R. Sloan, "Porosity evaluation of in-service thermal barrier coated turbine blades using a microwave nondestructive technique," NDT \& $E$ International, vol. 93, pp. 64-77, 2018.

[9] L. Tinsley, C. Chalk, J. Nicholls, J. Mehnen, and R. Roy, “A study of pulsed thermography for life assessment of thin EBPVD TBCs undergoing oxidation ageing," NDT \& E International, vol. 92, pp. 67-74, 2017.

[10] T. Kakuda, A. Limarga, A. Vaidya, A. Kulkarni, and T. D. Bennett, "Non-destructive thermal property measurements of an APS TBC on an intact turbine blade," Surface and Coatings Technology, vol. 205, no. 2, pp. 446-451, 2010.

[11] D. Ye, W. Wang, H. Zhou et al., "Characterization of thermal barrier coatings microstructural features using terahertz spectroscopy," Surface and Coatings Technology, vol. 394, Article ID 125836, 2020.

[12] W. Zhu, X. N. Cai, L. Yang, J. Xia, Y. C. Zhou, and Z. P. Pi, "The evolution of pores in thermal barrier coatings under volcanic ash corrosion using X-ray computed tomography," Surface and Coatings Technology, vol. 357, pp. 372-378, 2019.

[13] D. Ye, W. Wang, H. Zhou et al., "Quantitative determination of porosity in thermal barrier coatings using terahertz reflectance spectrum: case study of atmospheric plasma sprayed YSZ coatings," IEEE Transactions on Terahertz Science and Technology, vol. 10, no. 4, 2020.

[14] Y. Zhao, L. Lin, X. Li, and M. K. Lei, "Simultaneous determination of the coating thickness and its longitudinal velocity by ultrasonic nondestructive method," NDT \& E International, vol. 43, pp. 579-585, 2010.

[15] S. Lemlikchi, J. Martinsson, A. Hamrit, H. Djelouah, M. Asmani, and J. Carlson, "Ultrasonic characterization of thermally sprayed coatings," Journal of Thermal Spray Technology, vol. 28, no. 3, pp. 391-404, 2019.

[16] L. Wang, K.-Y. Ding, X.-P. Lin, Z. Li, R.-G. Zheng, and L.-W. Yang, "Defect evolution and microcracks of $8 Y S Z$ double-layer thermal barrier coatings by water immersion ultrasound macroscopic detection," Journal of Inorganic Materials, vol. 34, no. 12, pp. 1265-1271, 2019.

[17] B. Rogé, A. Fahr, J. Giguere, and K. I. McRae, "Nondestructive measurement of porosity in thermal barrier coatings," Journal of Thermal Spray Technology, vol. 12, pp. 530-535, 2003. 
[18] Y. Zhao, Z. Y. Ma, L. Lin, X. M. Li, and M. K. Lei, "Correlating ultrasonic velocity and porosity using FDTD method based on random pores model," Materials Science Forum, vol. 675-677, pp. 1221-1224, 2011.

[19] L. Lin, Y. Zhao, J. Chen, X. M. Li, and M. K. Lei, "Ultrasonic characterization of EB-PVD thermal barrier coatings irradiated by HIPIB," Key Engineering Materials, vol. 373-374, pp. 358-362, 2008.

[20] A. Fahr, J. S. R. Giguere, B. Roge, and K. I. McRae, "Application of NDE in aerospace coatings," in Smart Nondestructive Evaluation for Health Monitoring of Structural and Biological Systems, T. Kundu, Ed., pp. 83-92, SPIE-International Society for Optical Engineering, Bellingham, WA, USA, 2002.

[21] A. Mardanshahi, V. Nasir, S. Kazemirad, and M. M. Shokrieh, "Detection and classification of matrix cracking in laminated composites using guided wave propagation and artificial neural networks," Composite Structures, vol. 246, p. 112403 , 2020.

[22] O. I. Abiodun, A. Jantan, A. E. Omolara, K. V. Dada, N. A. Mohamed, and H. Arshad, "State-of-the-art in artificial neural network applications: a survey," Heliyon, vol. 4, Article ID e00938, 2018.

[23] R. Hecht-Nielsen, "Theory of the backpropagation neural network * based on "nonindent" by Robert Hecht-Nielsen, which appeared in proceedings of the international joint conference on neural networks 1, 593-611, june 1989, 1989 IEEE," in Neural Networks for Perception, pp. 65-93, Elsevier, Amsterdam, Netherlands, 1992.

[24] A. Khashman and P. Akpinar, "Non-destructive prediction of concrete compressive strength using neural networks," Procedia Computer Science, vol. 108, pp. 2358-2362, 2017.

[25] R. M. Galagan and A. S. Momot, "Analysis of application of neural networks to improve the reliability of active thermal NDT,” KPI Science News, vol. 133, no. 1, pp. 7-14, 2019.

[26] Y. Xu and R. Jin, "Measurement of reinforcement corrosion in concrete adopting ultrasonic tests and artificial neural network," Construction and Building Materials, vol. 177, pp. 125-133, 2018.

[27] L.-C. Shi, Q.-F. Zhao, Y. Long, and Y.-Z. Wang, "A detection method for porosity of gas turbine blade coating based on gray gradient space histogram entropy," in Proceedings of the 2018 International Conference on Audio, Language and Image Processing (ICALIP), pp. 22-27, IEEE, Shanghai, China, July 2018.

[28] D. Ye, W. Wang, Z. Xu, C. Yin, H. Zhou, and Y. Li, "Prediction of thermal barrier coatings microstructural features based on support vector machine optimized by cuckoo search algorithm," Coatings, vol. 10, no. 7, p. 704, 2020.

[29] D. Ye, W. Wang, H. Zhou et al., "Characterization of thermal barrier coatings microstructural features using terahertz spectroscopy," Surface and Coatings Technology, vol. 394, p. 125836, 2020.

[30] T. Lähivaara, L. Kärkkäinen, J. M. J. Huttunen, and J. S. Hesthaven, "Deep convolutional neural networks for estimating porous material parameters with ultrasound tomography," The Journal of the Acoustical Society of America, vol. 143, no. 2, pp. 1148-1158, 2018.

[31] Z. Ma, W. Zhang, Z. Luo, X. Sun, Z. Li, and L. Lin, "Ultrasonic characterization of thermal barrier coatings porosity through BP neural network optimizing Gaussian process regression algorithm," Ultrasonics, vol. 100, p. 105981, 2020.

[32] L. Lin, W. Zhang, Z. Ma, and M. Lei, "Porosity estimation of abradable seal coating with an optimized support vector regression model based on multi-scale ultrasonic attenuation coefficient," NDT \& E International, vol. 113, p. 102272, 2020.

[33] Z. Ma, Z. Luo, L. Lin, S. Krishnaswamy, and M. K. Lei, "Quantitative characterization of the interfacial roughness and thickness of inhomogeneous coatings based on ultrasonic reflection coefficient phase spectrum," NDT \& E International, vol. 102, pp. 16-25, 2019.

[34] H.-S. Zhang, F.-C. Wang, Z. Ma, Z.-F. Cheng, Q.-S. Wang, and W.-F. Xian, "Quantitative analysis of pores in plasmasprayed $\mathrm{ZrO}$ 2 2 coatings," Journal of Materials Engineering, vol. S1, 2006.

[35] Z.-Y. Ma, Z.-B. Luo, and L. Lin, "Characterization of ultrasonic longitudinal velocity evolution in TBCs with different porosity and void morphology based on random void model," Journal of Materials Engineering, vol. 4, pp. 86-90, 2014.

[36] Z. Ma, W. Zhang, Z. Luo, X. Sun, Z. Li, and L. J. U. Lin, "Ultrasonic characterization of thermal barrier coatings porosity through BP neural network optimizing," Gaussian Process Regression Algorithm, vol. 100, p. 105981, 2020.

[37] S. Zhang, Z. Ma, L. Lin, and W. Feng, "Comprehensive analysis for the effect of thermal barrier coating porosity on ultrasonic longitudinal wave velocity," in Proceedings of the Recent Featured Applications of Artificial Intelligence Methods LSMS 2020 and ICSEE 2020 Workshops, pp. 349-357, Springer, Shanghai, China, October 2020.

[38] G. Chen, K. Fu, Z. Liang et al., "The genetic algorithm based back propagation neural network for MMP prediction in CO2-EOR process," Fuel, vol. 126, pp. 202-212, 2014

[39] Y. Rong, Z. Zhang, G. Zhang et al., "Parameters optimization of laser brazing in crimping butt using Taguchi and BPNNGA," Optics and Lasers in Engineering, vol. 67, pp. 94-104, 2015.

[40] H. Liu, X. Qin, S. Huang, L. Jin, Y. Wang, and K. Lei, "Geometry characteristics prediction of single track cladding deposited by high power diode laser based on genetic algorithm and neural network," International Journal of Precision Engineering and Manufacturing, vol. 19, no. 7, pp. 1061-1070, 2018.

[41] F. Burden and D. Winkler, "Bayesian regularization of neural networks," in Artificial Neural Networks, pp. 23-42, Humana Press, Totowa, NJ, USA, 2008.

[42] S. Zhang, "Wind power prediction based on genetic neural network," AIP Conference Proceedings, vol. 1834, Article ID 020012, 2017.

[43] F. Yan and Z. Lin, "New strategy for anchorage reliability assessment of GFRP bars to concrete using hybrid artificial neural network with genetic algorithm," Composites Part B: Engineering, vol. 92, pp. 420-433, 2016.

[44] F. Zhang, Y. M. Fadul Mukhtar, B. Liu, and J. Li, "Application of ANN to predict the apparent viscosity of waxy crude oil," Fuel, vol. 254, p. 115669, 2019.

[45] W. Sun, M. Ye, and Y. Xu, "Study of carbon dioxide emissions prediction in Hebei Province, China using a BPNN based on GA," Journal of Renewable Sustainable Energy, vol. 8, Article ID 043101, 2016. 